\title{
Postural Stability Analysis with Inertial Measurement Units in Alzheimer's Disease
}

\author{
Miguel F. Gago ${ }^{a-c}$ Vitor Fernandes ${ }^{d}$ Jaime Ferreira ${ }^{d}$ Hélder Silvad \\ Luís Rochad $^{d}$ Estela Bichod Nuno Sousab, ${ }^{\text {b }}$ \\ a Neurology Department, Centro Hospitalar do Alto Ave, EPE, Guimarães, and \\ ${ }^{b}$ Life and Health Sciences Research Institute (ICVS), School of Health Sciences, \\ University of Minho, 'ICVS/3B's, PT Government Associate Laboratory, Braga/Guimarães, \\ and ${ }^{d}$ Center ALGORITMI, School of Engineering, University of Minho, Braga, Portugal
}

\section{Key Words}

Alzheimer's disease · Postural stability · Inclined surface $\cdot$ Inertial measurement unit · Triaxial accelerometer and gyroscope

\section{Abstract}

Background: The cause of frequent falls in patients with Alzheimer's disease (AD) is still not well understood. Nevertheless, balance control and sensory organization are known to be critical for moving safely and adapting to the environment. Methods: We evaluated postural stability in 20 AD patients (11 fallers and 9 nonfallers) and 16 healthy controls with an inertial measurement unit (triaxial accelerometers and gyroscopes) attached to the center of mass (COM) in different balance conditions (Romberg on flat surface and frontward/backwardinclined surface, with or without visual suppression) in a motor lab. Results: In AD patients, the group of fallers showed a different kinetic pattern of postural stability characterized by higher vulnerability to visual suppression, higher total/maximal displacement and a mediolateral/anteroposterior range of sway, and a consequent need for more corrections of COM pitch and roll angles. Conclusion: Further studies are needed to consolidate the normative values of the discriminatory kinetic variables with the potential of inclusion in a multifactorial analysis of the risk of falls. Nevertheless, these results highlight signs of impairment of central postural control in $A D$, which may require early therapeutic intervention. 


\section{Introduction}

Alzheimer's disease (AD) is the major cause of dementia in the geriatric population in the United States and Western Europe [1]. It is a neurodegenerative cortical disorder associated with posture and gait disturbances and a high risk of falls [2]. In AD patients, falls are more frequent and have more serious traumatic consequences, including hip fracture, than in nondemented elderly people $[3,4]$.

The underlying mechanisms contributing to falls in AD patients are not clearly understood [5]. Physiological deficits, such as impairment of sensorimotor function, reduced vision [6], peripheral sensory loss and muscle weakness [7] and slowed reaction time [8], either individually or globally [9], detected by a Physiological Profile Assessment test [10], may explain the higher risk of falls in AD. Also, the great variability in gait patterns [11] and increased postural sway [12] may account for this higher risk of falls in AD.

Most postural kinetics studies in $\mathrm{AD}$ are based on postural analysis of the center of pressure on force plates. In contrast, inertial measurement units (IMU), with integrated accelerometers and gyroscopes, are small, fully portable devices that are independent of the inclination in space and have proved to be equivalent to force platforms in the measurement of the center of mass (COM) and stability analysis [11-13]. Also, IMU have the advantage of measuring postural stability in several stability and environment scenarios, including inclined surfaces.

Herein, we aim to (1) analyze postural kinetics with IMU in AD patients and healthy controls in different postural stress conditions, such as Romberg, visual suppression, and inclination and (2) try to identify discriminative kinetic parameters in AD patients that may be predictors of falls.

\section{Methods}

\section{Subject Selection and Clinical Assessment}

The study population was recruited from our hospital outpatient neurology department. Patients with probable AD, according to Diagnostic and Statistical Manual of Mental DisordersIV (DSM-IV) and National Institute of Neurological and Communicative Disorders and Stroke/ Alzheimer's Disease and Related Disorders Association (NINCDS/ADRDA) criteria [13], were consecutively recruited for the study. The control group included age-matched caregivers of patients that had no history of falls or of neurological or psychiatric disease. Patients or controls were also excluded if there was a history of orthopedic, musculoskeletal or vestibular disorder or alcohol abuse. Demographic data and medical history were collected in both groups. A brief neuropsychological examination was performed using the Portuguese version of the Montreal Cognitive Assessment test (MoCA) with scores normalized to the Portuguese population [14], no more than 1 month prior to the kinetic assessment. Levels of education were categorized by years of schooling: 0 (analphabetic), 1 (1-4 years), 2 (5-9 years), 3 (10-12 years), and 4 (>12 years). The severity of dementia was graded according to the Clinical Dementia Rating (CDR) [15]. AD patients were recorded as fallers (ADF) if they had at least one fall in the previous 6 months. Written consent was obtained from all subjects or their legal guardians, and the study protocol was approved by the local ethics committee.

\section{Kinetic Postural Acquisition and Assessment}

Biometric data [weight, height, body mass index, and anthropometric measurements, i.e. shank (ankle-knee) and thigh (knee-iliac crest)] were collected on the day of kinetic postural assessment. Five kinetic sensing modules, harboring an 8051 microprocessor embedded in 
CC2530 Texas Instruments SoC (System on Chip) and an IMU MPU6000 (triaxial accelerometer and gyroscope) $[16,17]$ and operating with a sample rate frequency of $113 \mathrm{~Hz}$ on an SD card, were attached by Velcro bands to five body segments: trunk (COM; at 55\% of a person's height above the ground) [18], both legs (middle of ankle-knee distance), and both thighs (middle of knee-iliac crest distance). The video capture (sample rate of $60 \mathrm{fps}$ ) and the data logging on the five kinetic sensors were synchronized by bidirectional radio signal transmission by an USB coordinator node (connected to a PC with custom-made Matlab software). Outputs from the accelerometers were filtered with a second order Butterworth low-pass filter with a cutoff frequency of $0.5 \mathrm{~Hz}$ [19], and the outputs from the gyroscopes filtered with a cutoff frequency of $5 \mathrm{~Hz}$ [20].

Final pitch and roll angles were obtained by a complementary filter of accelerometer and gyroscope pitch and roll ( $\beta$-coefficient of 0.98) [21].

$$
\begin{aligned}
& \text { Pitch }(\theta)=\beta \cdot\left(\text { pitch }(\theta)_{\text {gyro }}\right)+(1-\beta) \cdot \operatorname{pitch}(\theta)_{\text {accel }} \\
& \text { Roll }(\phi)=\beta \cdot\left(\operatorname{roll}(\phi)_{\text {gyro }}\right)+(1-\beta) \cdot \operatorname{roll}(\phi)_{\text {accel }}
\end{aligned}
$$

The kinetic sensor orientation in space was calculated by Euler angle spatial representation for pitch $(\theta)$ and roll $(\phi)[22,23]$. After definitions of angles, displacement $(d)$ of the $\operatorname{COM}\left(H_{\text {Сом}}\right.$; i.e. $55 \%$ of a subject's height) was calculated with the formula:

$$
d y=\sin (\text { pitch }(\theta)) \cdot H_{\text {Сом} ;} d x=\sin (\operatorname{roll}(\phi)) \cdot H_{\text {Сом }} .
$$

One of a normal human's mechanisms of maintaining balance is to vary COM by bending knees and trunk. Therefore, the height of COM was constantly adjusted, using the information derived from the length $(L)$ of the shank and thigh (i.e. $L_{\text {shank }}$ and $L_{\text {thigh }}$, respectively) and from the angles of the IMU located on the shank and thigh (i.e. $\theta_{\text {shank }}$ and $\theta_{\text {thigh }}$, respectively) by the formula:

$$
\begin{aligned}
& T_{1}=\cos \theta_{\text {shank }} \cdot L_{\text {shank }} \\
& T_{2}=\cos \theta_{\text {thigh }} \cdot L_{\text {thigh }} \\
& H_{\text {COM }}=H_{\text {COM }}^{\text {measured }}-\left(L_{\text {shank }}-T_{1}\right)-\left(L_{\text {thigh }}-T_{2}\right) .
\end{aligned}
$$

From the kinetic measurements derived from the COM displacement, we focused on some that emerged from a systematic review as predictors of falls among elderly people [24]: total displacement $(\mathrm{cm})$ on the transverse plane

$$
\sqrt{\left(x_{i+1}-x_{i}\right)^{2}+\left(y_{i+1}-y_{i}\right)^{2}}
$$

maximal displacement $(\mathrm{cm})$ with respect to the origin on the transverse plane

$$
\text { maximum of } \sqrt{x_{i}^{2}+y_{i}^{2}} \text {; }
$$

maximal linear velocity $(\mathrm{cm} / \mathrm{s})[20]$; positioning $(\mathrm{cm})$ on $\mathrm{x}$ - and $\mathrm{y}$-axis (mean and range); roll angle (degrees; maximal, minimum, and mean), and pitch angle (degrees; maximal, minimum, and mean).

\section{Test Conditions}

Subjects were instructed to perform six different standing Romberg conditions: Romberg test with eyes open/closed on a flat firm surface and Romberg test on a backward/frontwardinclined surface with eyes open/closed. Subjects performed the Romberg test barefoot, with the medial aspects of the feet touching each other. During the tasks, the subjects stood quietly, with their arms hanging at their sides and their head in a normal forward-looking eye position with the eyes directed to an object $2 \mathrm{~m}$ away. All tasks were explained, and subjects had the opportunity to practice before the definitive trial. Each task was performed during $30 \mathrm{~s}$, and 
Gago et al.: Postural Stability Analysis with Inertial Measurement Units in Alzheimer's Disease

Table 1. Demographic, clinical, and anthropometric data of controls and AD patients (ADNF and ADF)

\begin{tabular}{|c|c|c|c|c|c|}
\hline & \multirow{2}{*}{$\begin{array}{l}\text { Controls } \\
(n=16)\end{array}$} & \multicolumn{2}{|l|}{ AD patients } & \multirow{2}{*}{$\begin{array}{l}\text { Intergroup } \\
\text { comparison }\end{array}$} & \multirow[t]{2}{*}{$\mathrm{p}$} \\
\hline & & $\operatorname{ADNF}(\mathrm{n}=9)$ & $\operatorname{ADF}(n=11)$ & & \\
\hline Females/males, n & $6 / 10$ & $7 / 2$ & $7 / 4$ & $\chi^{2}=4.02$ & 0.095 \\
\hline Age, years & $72.31 \pm 7.08$ & $73.56 \pm 8.72$ & $77.64 \pm 4.80$ & $\chi_{\mathrm{KW}}^{2}(2)=3.77$ & 0.152 \\
\hline Level of education ${ }^{1}$ & $1(0,4)$ & $1(0,2)$ & $1(0,2)$ & $\chi^{2}$ кw $(2)=4.383$ & 0.110 \\
\hline Height, m & $1.60 \pm 0.11$ & $1.52 \pm 0.06$ & $1.53 \pm 0.08$ & $\chi_{\mathrm{Kw}}^{2}(2)=5.208$ & 0.074 \\
\hline Weight, kg & $71.68 \pm 9.07$ & $68.90 \pm 9.82$ & $65.01 \pm 7.84$ & $\chi_{\text {кw }}^{2}(2)=4.084$ & 0.130 \\
\hline Body mass index & $27.92 \pm 3.44$ & $29.88 \pm 5.15$ & $27.80 \pm 2.84$ & $\chi^{2}$ кw $(2)=0.572$ & 0.751 \\
\hline $\mathrm{COM}, \mathrm{cm}$ & $88.27 \pm 6.04$ & $83.99 \pm 3.33$ & $84.15 \pm 4.29$ & $\chi^{2}{ }_{\mathrm{KW}}(2)=5.208$ & 0.074 \\
\hline \multirow{2}{*}{$\begin{array}{l}\text { Duration of disease, years } \\
\text { CDR }^{1}\end{array}$} & - & $3.33 \pm 1.94$ & $2.82 \pm 1.47$ & $\mathrm{U}=38.0 ; \mathrm{z}=-0.906$ & 0.407 \\
\hline & - & $1(0.5,2)$ & $2(0.5,2)$ & $\mathrm{U}=43.5 ; \mathrm{z}=-0.490$ & 0.726 \\
\hline \multirow[t]{4}{*}{ MoCA } & $24.75 \pm 3.59$ & $12.22 \pm 6.63$ & $10.09 \pm 4.42$ & $\chi_{\mathrm{KW}}^{2}(2)=24.023$ & $<0.001$ \\
\hline & & & & C vs. ADNF & $<0.001$ \\
\hline & & & & C vs. ADF & $<0.001$ \\
\hline & & & & ADF vs. ADNF & 0.643 \\
\hline
\end{tabular}

Unless otherwise indicated values represent mean \pm SD. C $=$ Controls.

${ }^{1}$ Values in parentheses represent minimum and maximum.

during that time the kinetic data were recorded $[25,26]$. The trial was invalidated and started again if subjects moved any part of their body, spoke, opened their eyes for visual aid or did a corrective step.

We set up a fixed $15^{\circ}$ inclined platform to standardly compare the adjustments of posture under inclination between the 3 groups. In our laboratory, on experiments of steps of $5^{\circ}$ of inclination, healthy subjects started to have a significant change on kinetic measurements after $15^{\circ}$ of inclination, being approximately the $20^{\circ}$ proposed by other studies [27]. The subject were in the Romberg position, with heels below toes for the task with the backwardinclined platform, and on the same inclined platform, with toes below heels for the Romberg task with the frontward-inclined platform. They rested between test conditions to reduce the effect of muscular fatigue, especially with platform tasks [28].

\section{Statistical Analysis}

Gender comparisons were analyzed by the $\chi^{2}$ Fisher exact test. Due to the absence of normality and variance equality amongst groups regarding continuous variables (anthropometrics, MoCA, years of disease, and kinetics parameters) and ordinal variables (education and CDR), the comparison between the groups was carried out by a nonparametric test, the Kruskal-Wallis test (comparison between 3 groups), with a pairwise post hoc analysis with Dunn's test and the magnitude of change in intraindividual tasks by the Wilcoxon matched pair test. Correlation analyses of age, anthropometrics, CDR and years of disease, with kinetic data, were performed with the Spearman test. All statistical analyses were conducted with statistical analysis software (SPSS 20.0) using a 95\% level of significance.

\section{Results}

\section{Demographic, Clinical, and Anthropometric Data}

This study included $20 \mathrm{AD}$ patients [9 classified as nonfallers (ADNF) and 11 as ADF] and 16 controls. There were no statistically significant differences between the groups, regarding age or anthropometric parameters (table 1). In spite of a higher frequency of females in the 
Dementia

Cognitive Disorders

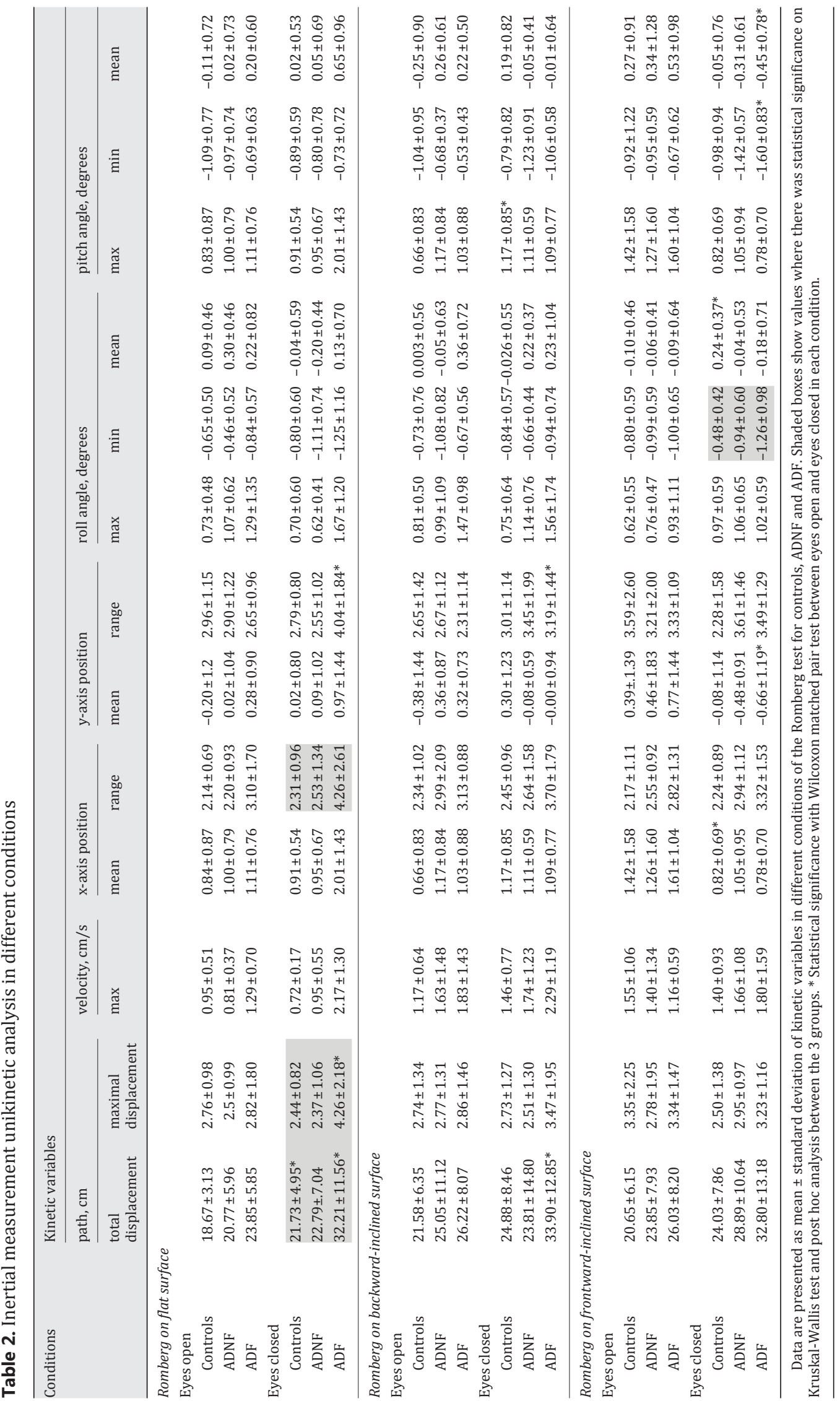

Gago et al.: Postural Stability Analysis with Inertial Measurement Units in Alzheimer's

Gago et

(C) 2014 S. Karger AG, Base
www.karger.com/dee 
Gago et al.: Postural Stability Analysis with Inertial Measurement Units in Alzheimer's Disease

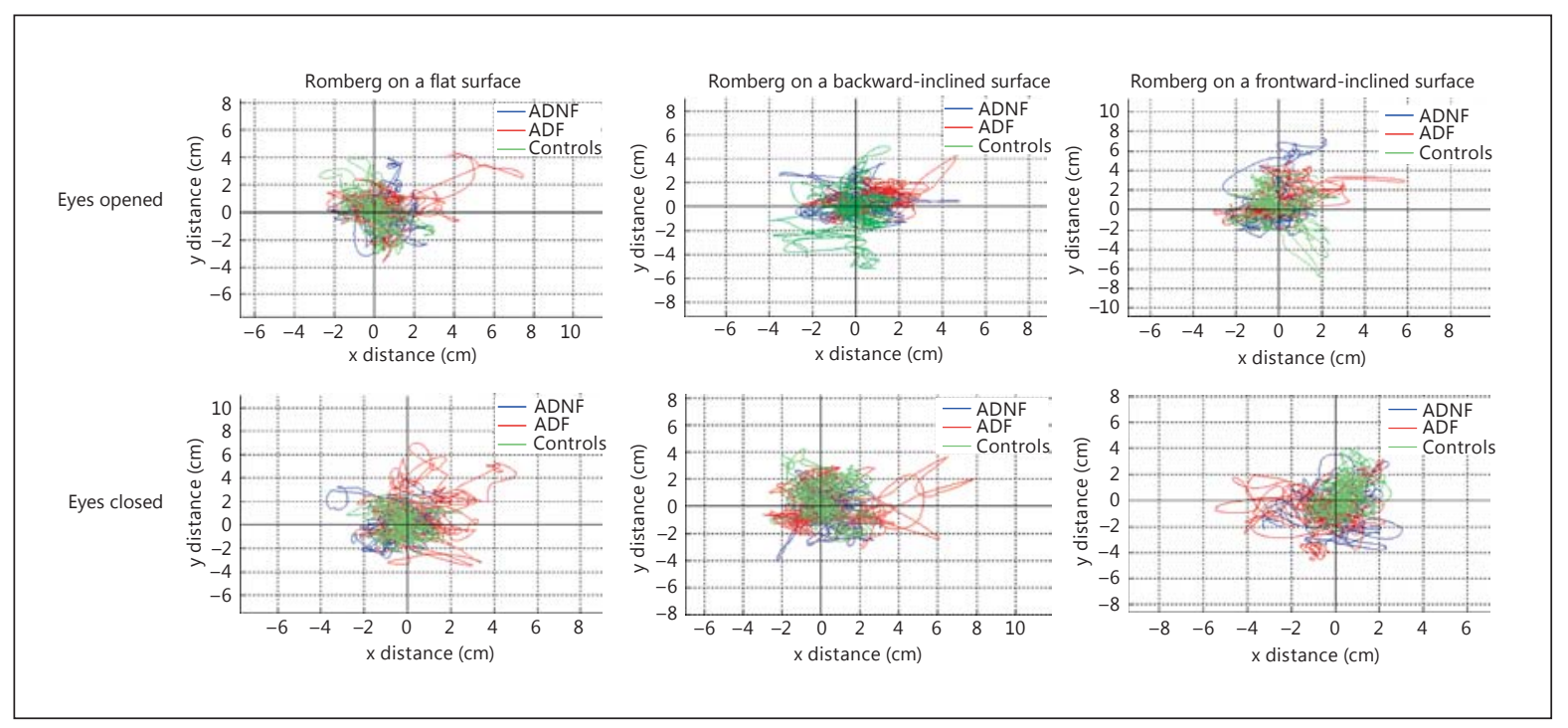

Fig. 1. Displacement plot on the $x$-axis (mediolateral) and the y-axis (anteroposterior) of the IMU, attached to the COM (at 55\% of height), for all subjects (controls, ADF, and ADNF).

AD patients, no statistical difference was detected regarding gender between the controls and ADF and ADNF patients. Moreover, the gender has not been associated with postural sway deficits [27]. The majority of AD patients and controls had an education level of less than 4 years. As expected, AD patients had lower scores on MoCA compared to controls, but there were no differences between ADNF and ADF in the total MoCA score or its subitems. ADF patients did not differ from ADNF patients regarding years of progression or severity of AD. No correlations were found between age, CDR, or years of disease with any of the kinetic variables.

\section{Kinetic Postural Analysis}

Romberg Position with Eyes Open/Eyes Closed on Flat Surface

In the Romberg position with eyes open, the kinetic posture measurements were not statistically different between the 3 groups. With eyes closed, the groups differed in the following kinetic posture measurements: total displacement $\left[\chi^{2}{ }_{\mathrm{KW}}(2)=6.608 ; \mathrm{p}=0.037\right.$; controls vs. ADF $\mathrm{p}=0.01$; controls vs. ADNF $\mathrm{p}=0.447$; ADF vs. ADNF $\mathrm{p}=0.127]$, maximal displacement $\left[\chi^{2}{ }_{\mathrm{KW}}(2)=9.241 ; \mathrm{p}=0.01\right.$; controls vs. ADF $\mathrm{p}=0.013$; controls vs. ADNF $\mathrm{p}=$ 0.948; ADF vs. ADNF $p=0.005]$, and range on $x$-axis $\left[\chi^{2}{ }_{\mathrm{KW}}(2)=9.036 ; p=0.01\right.$; controls vs. ADF $p=0.003$; controls vs. ADNF $p=0.645$; ADF vs. ADNF $p=0.034$ ] (table 2). The Wilcoxon matched pair test was used to compare between conditions with eyes open versus eyes closed, revealing that on visual suppression, controls had a statistically significant increase in total displacement $(\mathrm{Z}=-2.689 ; \mathrm{p}=0.005)$ and the ADF group had a statistically significant increase in total displacement $(Z=-2.490 ; p=0.01)$, maximal distance $(Z=-1.956 ; p=0.054)$, and range on $y$-axis $(\mathrm{Z}=-2.134 ; \mathrm{p}=0.032)$ (fig. 1$)$.

Romberg Position with Eyes Open/Eyes Closed on Inclined Platform

On the backward platform, no differences were found between the groups. On the frontward platform, with eyes closed, the AD patients, in particular the ADF patients, had a lower minimal roll angle $\left[\chi^{2}{ }_{\mathrm{KW}}(2)=10.442 ; \mathrm{p}=0.005\right.$; controls vs. ADF $\mathrm{p}=0.002$; controls vs. ADNF $\mathrm{p}=0.468 ; \mathrm{ADF}$ vs. ADNF $\mathrm{p}=0.044]$ (table 2). 
The Wilcoxon matched pair test was used to compare between conditions with eyes open and eyes closed. On the backward-inclined surface, under visual suppression, the controls had an increase in maximal pitch angle $(\mathrm{Z}=-2.223$; $\mathrm{p}=0.025)$ towards anterior (positive values) inclination, and the ADF patients had an increase in total displacement $(\mathrm{Z}=-2.599$; $\mathrm{p}=0.006)$ and the range on the $\mathrm{y}$-axis $(\mathrm{Z}=-2.395 ; \mathrm{p}=0.014)$.

On the frontward-inclined surface, under visual suppression, controls shifted to a lower mean $\mathrm{x}$-axis position $(\mathrm{Z}=-2.120 ; \mathrm{p}=0.034)$ and had an increase in the mean roll angle $(\mathrm{Z}=$ -2.223; $p=0.025)$. The ADF group had a significant change in the minimal pitch angle $(Z=$ -2.667; $p=0.005$ ) towards posterior inclination, almost reaching significance on a more posterior (negative) mean $\mathrm{y}$-axis position $(\mathrm{Z}=-1.956$; $\mathrm{p}=0.054$ ) and on a mean pitch angle $(Z=-1.956 ; p=0.054)$ (fig. 1).

\section{Discussion}

The cause of frequent falls in patients with $\mathrm{AD}$ is still not well understood. Balance control and sensory organization are known to be critical for moving safely and adapting to the environment. Herein, we have explored the underlying mechanisms for this tendency to fall and we have shown that ADF patients display a different kinetic profile.

Balance is a complex process of coordination of multiple body systems, including the vestibular, auditory, visual, motor and higher level premotor systems, that generate appropriate synergic postural muscle movements of the head, eye, trunk, and limbs to maintain posture [29]; this is achieved by sustaining, achieving, or restoring the body COM relative to the base of support or, more generally, within the limits of stability with a minimal sway [30]. An individual's limits of stability, commonly referred to as functional stability limits, refer to the maximum distance in which one can voluntarily displace one's center of gravity and lean one's body in a given direction without losing one's balance [31].

Visual suppression makes the human body more dependent on vestibular and proprioceptive systems, consequently increasing sway [9], which was confirmed in our study both in healthy and AD subjects. However, the visual suppression effect was stronger in ADF patients. After closing their eyes, the ADF group swayed more (total displacement) and beyond safety limits (maximal displacement). Contrary to controls that presented on visual suppression a normal correction acquiring a more central position (lower mean $\mathrm{x}$ - and $\mathrm{y}$-axis positions and range of sway), the ADF group had an increase in the range of mediolateral/anteroposterior sway. Our results agree with previous literature which has shown that the mediolateral sway is associated with a higher risk of falls in elderly people [24], and the anteroposterior sway is a discriminative parameter of AD versus controls [9] and also of fallers versus nonfallers in cognitively able older people [32]. This increased sway also demanded more pitch and roll variations, and ankle and trunk strategies of correction of stability [33] in the ADF group.

We also aimed to evaluate the susceptibility to inclination, as AD patients can walk long distances and thus are subjected to constant environmental postural stress, such as surface inclination, which may account for their falls [34]. Postural control on a tilting support surface is mainly achieved with the help of visual, vestibular, and proprioceptive afferents [27]. On a $20^{\circ}$ static inclined surface, the inclination was described to significantly increase postural imbalance in healthy subjects, especially when visual support was interrupted [27]. Contrary to what we had primarily expected, on the inclined surface, there was an attenuation of the differences between groups that were more evident on the flat surface. A learning trial repetition bias [35], an instruction anticipation factor, and a higher demand of attention and focus could have accounted for a better postural sway performance on the inclined surfaces in comparison to the less stressful flat surface condition. However, the controlled lab conditions 
are exactly the opposite of the daily living conditions where patients are more relaxed, unfocused, and without the anticipation effect of fear of falling [36] or preparation for episodes of disequilibrium. This clearly highlights the importance of cognition, especially attention, on postural balance [12], and the need for complementing lab analysis with kinetic studies on everyday motion behavior [37].

In a recent study, older adults with presumptive preclinical $\mathrm{AD}$, with higher levels of brain fibrillar amyloid plaques measured by Pittsburgh compound B retention on brain PET imaging, had a short latency time to their first fall [38]. This raises the hypothesis that neuropathological changes that negatively affect postural control and increase the risk of falls may happen subclinically in AD patients [32, 39, 40]. In fact, in our study, ADF and ADNF patients, although having different kinetic performances, were clinically very similar, not differing in age, anthropometric data, neuropsychological assessment, or severity of the disease. Therefore, kinetic postural analysis, in our study measured with IMU, may be a useful tool to preclinically identify AD patients with a higher risk of falls.

Although we need more studies to consolidate normative values of discriminatory kinetic variables acquired by IMU, with the potential of inclusion in a multifactorial analysis of the risk of falls, our results highlight signs of impairment of central postural control in AD, which may require early therapeutic intervention.

\section{Disclosure Statement}

The Center ALGORITMI was funded by the Neural Engineering Transformative Technologies (NETT) project.

\section{References}

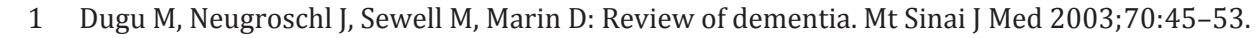

-2 Scarmeas N, Hadjigeorgiou GM, Papadimitriou A, Dubois B, Sarazin M, Brandt J, et al: Motor signs during the course of Alzheimer disease. Neurology 2004;63:975-982.

-3 Sato Y, Kanoko T, Satoh K, Iwamoto J: Risk factors for hip fracture among elderly patients with Alzheimer's disease. J Neurol Sci 2004;223:107-112.

4 Buchner DM, Larson EB: Falls and fractures in patients with Alzheimer-type dementia. JAMA 1987;257:14921495.

5 Morris JC, Rubin EH, Morris EJ, Mandel SA: Senile dementia of the Alzheimer's type: an important risk factor for serious falls. J Gerontol 1987;42:412-417.

6 Uhlmann RF, Larson EB, Koepsell TD, Rees TS, Duckert LG: Visual impairment and cognitive dysfunction in Alzheimer's disease. J Gen Intern Med 1991;6:126-132.

7 Moreland JD, Richardson JA, Goldsmith CH, Clase CM: Muscle weakness and falls in older adults: a systematic review and meta-analysis. J Am Geriatr Soc 2004;52:1121-1129.

-8 Gordon B, Carson K: The basis for choice reaction time slowing in Alzheimer's disease. Brain Cogn 1990;13: 148-166.

9 Leandri M, Cammisuli S, Cammarata S, Baratto L, Campbell J, Simonini M, et al: Balance features in Alzheimer's disease and amnestic mild cognitive impairment. J Alzheimers Dis 2009;16:113-120.

10 Lorbach ER, Webster KE, Menz HB, Wittwer JE, Merory JR: Physiological falls risk assessment in older people with Alzheimer's disease. Dement Geriatr Cogn Disord 2007;24:260-265.

11 Webster KE, Merory JR, Wittwer JE: Gait variability in community dwelling adults with Alzheimer disease. Alzheimer Dis Assoc Disord 2006;20:37-40.

12 Manckoundia P, Pfitzenmeyer P, d'Athis P, Dubost V, Mourey F: Impact of cognitive task on the posture of elderly subjects with Alzheimer's disease compared to healthy elderly subjects. Mov Disord 2006;21:236241.

13 McKhann GM, Knopman DS, Chertkow H, Hyman BT, Jack CR Jr, Kawas CH, et al: The diagnosis of dementia due to Alzheimer's disease: recommendations from the National Institute on Aging-Alzheimer's Association workgroups on diagnostic guidelines for Alzheimer's disease. Alzheimers Dement 2011;7:263-269.

14 Freitas S, Simoes MR, Alves L, Santana I: Montreal Cognitive Assessment (MoCA): normative study for the Portuguese population. J Clin Exp Neuropsychol 2011;33:989-996. 
Gago et al.: Postural Stability Analysis with Inertial Measurement Units in Alzheimer's Disease

15 Morris JC: The Clinical Dementia Rating (CDR): current version and scoring rules. Neurology 1993;43:24122414.

16 Nakayama N, Okumura A, Shinoda J, Yasokawa YT, Miwa K, Yoshimura SI, et al: Evidence for white matter disruption in traumatic brain injury without macroscopic lesions. J Neurol Neurosurg Psychiatry 2006;77: 850-855.

-17 Afonso JA, Silva HD, Macedo P, Rocha LA: An enhanced reservation-based MAC protocol for IEEE 802.15.4 networks. Sensors (Basel) 2011;11:3852-3873.

18 Winter DA, Patla AE, Frank JS: Assessment of balance control in humans. Med Prog Technol 1990;16:31-51.

19 Watanabe T, Saito H, Koike E, Nitta K: A preliminary test of measurement of joint angles and stride length with wireless inertial sensors for wearable gait evaluation system. Comput Intell Neurosci 2011;2011:975193.

20 Winter DA: Biomechanics and Motor Control of Human Movement, ed 4. Hoboken, Wiley, 2009, xiv, p 370.

-21 Roan P, Deshpande N, Wang Y, Pitzer B: Manipulator state estimation with low cost accelerometers and gyroscopes. IEEE/RSJ International Conference on Intelligent Robots and Systems, Vilamoura, 2012, pp 48224827.

22 Tsang C: Error Reduction Techniques for a MEMS Accelerometer-Based Digital Input Device. Hong Kong, The Chinese University of Hong Kong, 2008.

-23 Silva HR, Rocha LA, Afonso JA, Morim PC, Oliveira PM, Correia JH: Wireless hydrotherapy smart-suit network for posture monitoring. IEEE International Symposium on Industrial Electronics, Vigo, 2007, pp 2713-2717.

24 Piirtola M, Era P: Force platform measurements as predictors of falls among older people - a review. Gerontology 2006;52:1-16.

25 Carpenter MG, Frank JS, Winter DA, Peysar GW: Sampling duration effects on centre of pressure summary measures. Gait Posture 2001;13:35-40.

26 Le Clair K, Riach C: Postural stability measures: what to measure and for how long. Clin Biomech (Bristol, Avon) 1996;11:176-178.

27 Frames C, Soangra R, Lockhart TE: Assessment of postural stability using inertial measurement unit on inclined surfaces in healthy adults - Biomed 2013. Biomed Sci Instrum 2013;49:234-242.

-28 Nardone A, Tarantola J, Giordano A, Schieppati M: Fatigue effects on body balance. Electroencephalogr Clin Neurophysiol 1997;105:309-320.

29 Horak FB, Henry SM, Shumway-Cook A: Postural perturbations: new insights for treatment of balance disorders. Phys Ther 1997;77:517-533.

30 Pollock AS, Durward BR, Rowe PJ, Paul JP: What is balance? Clin Rehabil 2000;14:402-406.

-31 Patton JL, Pai Y, Lee WA: Evaluation of a model that determines the stability limits of dynamic balance. Gait Posture 1999;9:38-49.

-32 Merlo A, Zemp D, Zanda E, Rocchi S, Meroni F, Tettamanti M, et al: Postural stability and history of falls in cognitively able older adults: the Canton Ticino study. Gait Posture 2012;36:662-666.

33 Maeda Y, Tanaka T, Nakajima Y, Shimizu K: Analysis of postural adjustment responses to perturbation stimulus by surface tilts in the feet together position. J Med Biol Eng 2011;31:301-305.

34 Chong RK, Horak FB, Frank J, Kaye J: Sensory organization for balance: specific deficits in Alzheimer's but not in Parkinson's disease. J Gerontol A Biol Sci Med Sci 1999;54:M122-M128.

-35 Corriveau H, Hebert R, Prince F, Raiche M: Intrasession reliability of the 'center of pressure minus center of mass' variable of postural control in the healthy elderly. Arch Phys Med Rehabil 2000;81:45-48.

-36 Lajoie Y, Gallagher SP: Predicting falls within the elderly community: comparison of postural sway, reaction time, the Berg balance scale and the Activities-specific Balance Confidence (ABC) scale for comparing fallers and non-fallers. Arch Gerontol Geriatr 2004;38:11-26.

-37 Kirste T, Hoffmeyer A, Koldrack P, Bauer A, Schubert S, Schroder S, et al: Detecting the effect of Alzheimer's disease on everyday motion behavior. J Alzheimers Dis 2014;38:121-132.

-38 Stark SL, Roe CM, Grant EA, Hollingsworth H, Benzinger TL, Fagan AM, et al: Preclinical Alzheimer disease and risk of falls. Neurology 2013;81:437-443.

-39 Demura S, Kitabayashi T, Aoki H: Body-sway characteristics during a static upright posture in the elderly. Geriatr Gerontol Int 2008;8:188-197.

40 Demura S, Kitabayashi T, Kimura A, Matsuzawa J: Body sway characteristics during static upright posture in healthy and disordered elderly. J Physiol Anthropol Appl Human Sci 2005;24:551-555. 